\title{
ANÁLISIS DE LA INFLUENCIA DE LA FAMILIA EN LOS HÁBITOS LECTORES DE SUS HIJAS E HIJOS: UN ESTUDIO ETNOGRÁFICO
}

\author{
Emilia Moreno Sánchez \\ Universidad de Huelva
}

\begin{abstract}
RESUMEN. La lectura y su aprendizaje supone una de las preocupaciones de las familias, de los docentes y de todos los niños y las niñas en edad escolar, especialmente en su inicio, ya que de su desenvolvimiento en este aprendizaje dependerá el éxito o fracaso en su futuro escolar. Sin embargo, normalmente existe desconocimiento en cada contexto de lo que ocurre con la lectura en los otros. Así, los padres y madres ignoran qué es lo que pasa en el colegio, y los maestros y maestras no saben qué ocurre en los hogares de los niños. Ambos (familia y escuela) desconocen cómo favorecer mutuamente el desarrollo de la lectura en el ambiente natural, social y cultural en la infancia. Por ello, es necesario investigar acerca de los contextos en los que se realiza la lectura, especialmente en la familia, por la influencia tan temprana, constante y duradera que tiene, estudiando cuáles son los factores que van a favorecer o dificultar la lectura, y de cuyo balance resultarán los hábitos de lectura o su ausencia. Por otra parte, consideramos que, más que llevar a cabo encuestas para constatar si la gente lee, son necesarios trabajos que investiguen el verdadero trasfondo de la lectura. En esta idea nos hemos basado para realizar el estudio que se encuadra dentro de la etnografía descriptiva, empleando como estrategia el estudio de casos.
\end{abstract}

ABSTRACT. Reading and its learning is one of the main concerns for families, teachers and children, particularly in the first school yerars. Sucess or failure in further school levels depends on it. However, there is a great ignorance among all these contexts: parents ingnore what happens in the school and so do teachers about what happens at home. Both (school and family) don't collaborate in order to facilitate and improve the reading learning in a natural, social and culture enviroment. For this reason, it is necessary to make some research about the contexts in which reading is acquired. Specially, family context needs to be analysed because of its early, constant and permanent influence. Factors that facilitate or impede reading and that promote or not good reading habits must be identify. In other way, we also think that research on reading background is more important than survey studies about the reading habits of big samples. In such idea we have based on the ethnographic descriptive research presented in this article, in which we have used the case study strategy. The methodologic process developed and the results of this research are presented in this article.

\section{Introducción}

La familia es una institución universal, aunque su configuración funcional depende de la estructura y de la cultura de la sociedad en que se inserte. Esta circunstancia 
va a ocasionar que sus funciones adquieran más o menos preponderancia dependiendo del contexto geográfico e histórico. La diversidad funcional hace que, a su vez, aparezcan nuevas formas de composición (extensa, nuclear,...) y también nuevas formas de relación entre los miembros (papeles sexuales, autoridad, etc.).

Las funciones asignadas históricamente (educativas, religiosas, económicas, de control social, etc.) se han visto reducidas en la actualidad a las que son vitales para la superviviencia de la sociedad: la sexual, la reproductiva, la económica y la educativa. En este trabajo nos centraremos en esta última.

De todos los agentes de socialización, la familia es el más importante además del primero en presentarse $y$, en muchos casos, es el último en desaparecer. El medio familiar, constituye durante muchos años el lugar donde los niños y las niñas se desarrollan social y psicológicamente. Los factores sociales intervienen a través de la influencia de los padres y madres (sus comportamientos, deseos, lenguaje, actitudes,...), constituyendo junto con la escuela, posteriormente, "los delegados estructurales de la sociedad global junto al individuo" (Association Française pour la lecture, 1983:103). Las primeras normas, valores, modos de conducta los percibe el ser humano en el contexto familiar, conformándose de una manera bastante determinante la personalidad de las personas, la actitud y el comportamiento frente a la sociedad, en definitiva su desarrollo.

A pesar de la aparente espontaneidad e improvisación de las pautas educativas familiares, su organización es un complejo que obedece a una serie de mecanismos. En primer lugar, se encuentra el sistema de interacción en que descansa la vida familiar. El niño o la niña va incorporando a su personalidad hábitos, conductas, valores y sentimientos sociales mediante un intercambio de experiencias encauzadas en expectativas pautadas. Por otra parte está el sistema de recompensas y castigos. A través de ellos, de una forma consciente, esta institución, procura que se mantengan comportamientos y hábitos entre las personas que la componen.

Hay que subrayar también la importancia de los modelos de comportamiento. Este mecanismo, obedece muchas veces a procesos inconscientes, pero que son tan perdurables como cualquiera de los anteriores. Al hacerse mayores, sin embargo aparecen otros personajes interesantes en el círculo de amistades, y esta admiración que sentían hacia sus familiares cercanos ya no es exclusiva.

Estas influencias van a ser determinantes para el resto de su vida. Sin embargo no siempre se dan estos procesos de forma positiva, dependiendo de factores, como el clima emocional, los estilos educativos o la existencia de un entorno socialmente estimulante o la relación emocional entre los progenitores y sus hijos/as.

La relación que se establezca entre padres, madres con los niños y niñas va a condicionar no sólo sus reacciones inmediatas, sino también los aprendizajes posteriores. En este hecho demostrado, existen tres hitos fundamentales en el ámbito del aprendizaje temprano (Polaino-Lorente, 1997). El primero es que el modo en que las personas adultas quieren lo que enseñan va a condicionar que el niño o la niña quiera lo que aprende. En segundo lugar, el hecho de que el cuidador/a desee enseñar al niño y la niña puede seguirse del querer aprender. Por último, que es preciso que el niño/a se sienta querido/a por quien va a enseñarle. Estos tres hitos, interrelacionados van a condicionar una actitud de la que dependerán sus futuros aprendizajes. El apego establecido entre la madre o el padre y el niño/a va a repercutir en su desarrollo social, emocional y cognitivo. 
Las prácticas educativas de las familias, a su vez, están condicionadas por una serie de factores como el sexo, la edad, el orden de nacimiento, características de personalidad y nivel educativo de los padres. Así, cuanto mayor es el nivel de escolarización de los padres y madres más protagonistas se sienten de su desarrollo y sostienen expectativas de logro más altas (Palacios, 1988, cit. Cubero y Moreno, 1994). Coleman (1996) afirma que se puede demostrar experimentalmente cómo el tener padres y madres emocionalmente inteligentes tiene una repercusión positiva entre los hijos y las hijas. Sostiene que las lecciones emocionales (apego) pueden entremezclarse de forma natural, con la lectura, la escritura, la salud, etc. y, de esta forma, van a contribuir a un desarrollo armónico y a la formación de hábitos. Aunque estas diferencias, al igual que los estilos educativos, no son actitudes manifiestamente conscientes en la familia (Silvestre y Solé, 1993).

Además de los factores anteriores, hay otros aspectos relacionados con la familia y su ambiente que también van a influir en el desarrollo personal y social, como son las características de la vivienda. La circunstancia de que exista un contexto estimulante va a repercutir favorablemente en el desarrollo de los niños y las niñas. Aunque hay un hecho claro y es que el medio social puede ser o no estimulante en función de la presencia de unos factores, pero no coincide ni con las características socioeconómicas o socioculturales como se podría deducir de un análisis superficial de las clases desfavorecidas, sino de variables más actitudinales y afectivas.

Hay autores que afirman que el aprendizaje se va a producir en ausencia de estos factores o a pesar de la existencia de otros, y que su repercusión va a depender de las características individuales. Lo que sí parece claro es que el medio social debe estimular los aprendizajes. Concretamente, el medio estimulante en relación con los hábitos lectores es el de aquellas familias en las que se valora la lectura, aunque sólo sea porque se le narran cuentos o se le leen historias.

Si se considera que leer es un proceso complejo que consiste en saber descifrar, comprender lo que se lee, ser capaz de juzgar su contenido y de gustar de la lectura (Foucambert, 1989), en él no sólo van a intervenir factores cognitivos y lingüísticos, sino también ambientales, siendo estos últimos los que van a facilitar la adquisición de la lectura.

La familia es, en este sentido y especialmente en los primeros momentos del aprendizaje lector, quien ejerce una influencia más directa, entre otros aspectos, en la motivación por la lectura.

El placer por leer no se despierta de forma automática, sino que supone un aprendizaje, en el cual toda esta estimulación ambiental, no sólo debe estar disponible sino que debe adecuarse a las habilidades e intereses de los niños y niñas, encontrándose en esta adecuación el paso fundamental para generar la motivación necesaria para el aprendizaje lector y el gusto por la lectura. En este sentido, los mecanismos que posee la familia son muchos. Algunos de ellos ya los hemos señalado. Otros son, por ejemplo, el tipo de interacción que mantienen padres y madres con los hijos e hijas, lo que va a influir en la adquisición de habilidades lingüísticas y cognitivas necesarias para la lectura; o el modo en que los padres o madres acomodan su discurso a las posibilidades de comprensión de los niños y las niñas; el tiempo que los progenitores dedican a la lectura y la importancia que dan a ésta; el implicar al niño o la niña en actividades cotidianas como hacer la lista de la compra, etc. Se puede destacar una actividad que no por sencilla o conocida es menor su eficacia en este proceso motivacional, como es la narración de historias y cuentos por parte de la familia. 
Los trabajos que demuestran la influencia de las madres y los padres sobre los aprendizajes de los/as niños/as son muy numerosos (Palacios, Marchesi y Coll, 1991; Panchón Iglesias, 1995; Vygotsky, 1978; Bloomquist,1996). Sin embargo, específicamente en relación con la lectura son pocos los estudios llevados a cabo y fundamentalmente siguen una tradición anglosajona. Sobre la importancia que tienen los factores ambientales y específicamente los familiares en relación con los hábitos lectores hay una línea de investigación que nos aporta datos interesantes. En este sentido, en un estudio sobre los factores que podían fomentar el uso de la lectura en casa se consideraron como variables la clase social, situación laboral, hábitos lectores de las personas adultas, enfermedades, relaciones con el colegio y actitudes de madres y padres hacia la ayuda de sus hijos e hijas en casa (Weinberger, 1986). Se concluye que es decisiva la influencia de éstos factores en el hábito lector de las hijas e hijos. Este apoyo puede eliminar incluso las dificultades de aprendizaje, dislexia y los problemas de conducta mejorando la comprensión, la fluidez y el uso del contexto que pueden presentar los niños y las niñas. Esto también se ha puesto de manifiesto en un programa, que resalta la importancia de aprender con refuerzo positivo, con buenos ejemplos y prácticas positivas (Topping y Mcknight, 1984), resaltando este valor por encima de otras variables (Topping, 1986 y1987; Miller, 1986).

En un trabajo previo (Rodríguez, Moreno y Muñoz, 1988) expusimos la relación existente entre diversos aspectos del entorno familiar y medidas globales de habilidad lectora, destacando entre ellos el tipo de interacciones que mantienen los padres y las madres con los hijos e hijas, la cantidad de tiempo que pasan padre/madre con el niño o niña, los hábitos de los padres tales como la cantidad de lectura y el valor que otorga a la lectura y el hecho de proporcionar actividades enriquecedoras, tales como actividades culturales, viajes y la disponibilidad de materiales de lectura.

En este sentido, se puede pensar que los hábitos lectores, en tanto que actitudes y valores que las personas portan y moldean durante su vida, tienen su fundamento en la calidad o tipo de ambiente familiar en el que se han desarrollado estas personas durante la infancia. Si se consideran las actitudes como imitación, parece claro que si la madre y el padre son amantes de la lectura y leen habitualmente, lo más probable es que su hija/o mediante la imitación de las actitudes familiares las adopten como propias y también se interesen por la lectura. Sin embargo, no todas las influencias son positivas y de hecho hay otras conductas y actitudes, cuya existencia, en lugar de interesar a los niños y las niñas les desmotivan (Adán, 1992). Por ejemplo, dejar de contarles cuentos desde muy pronto, recriminarles continuamente con frases como "antes leías más" o culpar sólo a la televisión de su falta de interés por la lectura, cuando muchas veces la responsabilidad la tienen las personas adultas. Así, en el estudio realizado en nuestro contexto con una muestra representativa de chicas y chicos pertenecientes a colegios públicos de Málaga capital (Moreno, Padilla y Vélez, 1998) pudimos observar que un 36\% de los niños y niñas manifiestan que sus padres/madres les dicen que vean la televisión "a veces" (si sumamos este porcentaje al de la categoría "siempre", hallamos que casi un $40 \%$ de los sujetos son animados a ver la televisión). Afortunadamente, las personas encuestadas manifiestan, en un $79 \%$, que sus padres les animan a leer "siempre" o "a veces". De cualquier forma, estos hallazgos merecen especial atención porque parecen indicar que la familia no anima a sus hijos e hijas a leer tanto como sería necesario, lo que puede perjudicar grandemente la adquisición de los hábitos lectores y la actitud hacia la lectura (Gráfico 1). 


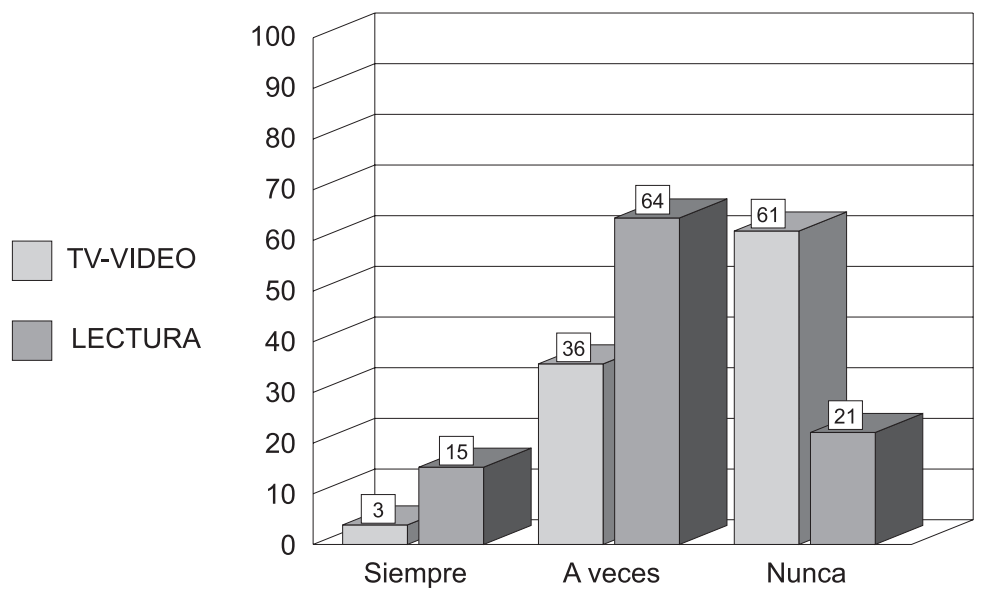

Gráfico 1: Frecuencia con la que los padres/madres animan a sus hijos/as a ver la televisión o el vídeo y a leer.

En definitiva, la lectura no debe estar alejada de la vida y son las madres y los padres el puente que enlaza con la escuela donde, aunque no exclusivamente, se da el aprendizaje de la lectura.

Parece ser que la lectura y su aprendizaje supone, una de las preocupaciones de las familias, de docentes y de todos los niños y las niñas en edad escolar, especialmente en su inicio, ya que de su desenvolvimiento en este aprendizaje, muy delicado, dependerá el éxito o fracaso en su futuro escolar.

Sin embargo, normalmente existe desconocimiento en cada contexto de lo que ocurre con la lectura en los otros. Así, los padres y madres ignoran qué es lo que pasa en el colegio, los maestros y maestras no saben qué ocurre en los hogares de los niños, ambos (familia y escuela) desconocen cómo favorecer mutuamente el desarrollo de la lectura en el ambiente natural social y cultural en la infancia. Un conocimiento mutuo, creemos, facilitaría el diálogo entre el profesorado y los padres y madres y además el aprendizaje y la adquisición de los hábitos lectores de los niños y niñas.

Por todo ello, es necesario investigar acerca de los contextos en los que se realiza la lectura, especialmente en la familia, estudiando cuáles son los factores que van a favorecer o dificultar la lectura y de cuyo balance resultarán los hábitos de lectura o su ausencia. Por otra parte, consideramos que más que llevar a cabo encuestas para constatar si la gente lee, son necesarios trabajos que investiguen el verdadero trasfondo de la lectura. En esta idea nos hemos basado para realizar el estudio que se presenta en este artículo.

\section{El proceso de la investigación}

Esta investigación se encuadra dentro de lo que se denomina etnografía descriptiva (Taylor y Bogdan, 1985) y como estrategia se ha empleado el estudio de casos. Por eso, se ha pretendido, por un lado proporcionar una imagen lo más fiel posible de la realidad, de lo que la gente dice y del modo en que actúa, y por otro, se trató de 
redactar los resultados e interpretar los hallazgos obtenidos, aunque somos conscientes de que será el lector o lectora quien deberá extraer sus propias conclusiones y generalizaciones a partir de los datos.

\subsection{Descripción del contexto}

Las familias pertenecen, fundamentalmente, a dos de las muchas barriadas que componen el extrarradio de Málaga capital, declaradas por la Administración como de especial problemática social.

Se trata de barriadas relativamente modernas. De unos 25 a 30 años de antigüedad. Sin dotación alguna de servicios públicos. En el inicio de su creación existió una fortísima especulación del suelo. En la actualidad apenas se han realizado edificaciones ni modificaciones en las ya existentes, salvo la construcción en una de las avenidas principales de una Guardería de Servicios Sociales. Los bloques de 14 y 15 plantas se encuentran prácticamente unos junto a otros sin apenas zonas verdes o ajardinadas, y en muchos casos, sin aceras, sin aparcamientos subterráneos, lo que le da un aspecto laberíntico. Solamente existe un pequeño parque de reciente construcción. En el barrio no hay espacios donde reunirse, ni bancos donde sentarse, por lo que se puede observar a las personas adultas (fundamentalmente hombres) charlando en grupos en las zonas cercanas a bares y cafeterías. La gente joven suele sentarse en las escaleras o permanecen de pie en las calles. En los bajos de los bloques existen numerosos bares, tiendas de comestibles, panaderías, carnicerías, tiendas de ropa, en definitiva comercio de consumo primario.

La mayoría de las viviendas existentes son lo que se denomina viviendas de promoción pública (VPP). Son pisos que se construyen para albergar a las familias más necesitadas, quienes deben pagar un alquiler simbólico o una pequeña cuota tras el abono de la cual se convierten en propietarias. Estas viviendas se mezclan con otras de protección oficial (VPO) más "normalizadas" (término empleado por las personas de los Servicios Sociales), lo que contribuye a la existencia de dos tipos de familias desde el punto de vista socioeconómico.

En cuanto a la población es típicamente de aluvión. Llegaron a Málaga atraídos por el «boom» turístico de los años 60. En un principio procedían, fundamentalmente, de los pueblos de la provincia. En la actualidad el 76,1\% ha nacido en Málaga capital, observándose un descenso en las personas nacidas en la provincia entre 1991 y 1995.

Sus habitantes tienen en común que son jóvenes con alta tasa de natalidad. Aproximadamente 3,5 hijos por familia. Es mano de obra sin cualificar y, hoy se encuentran con las lacras del paro y la multiplicación de los casos de prostitución, alcoholismo, drogodependencia, delincuencia, etc. alcanzando trágicas cifras.

En general, sus hijos/as no se encuentran motivados culturalmente por el ambiente en el que se ven inmersos/as. Lo que se une a las tensiones familiares y al desinterés hacia el estudio. Esto origina en las aulas frecuentes problemas de disciplina por lo que las Ilamadas a los padres y madres por estas causas son habituales.

\subsection{La muestra}

La selección de la muestra estaba sujeta a las premisas establecidas en el estudio. Así, para que esta investigación tuviera sentido y no aportara exclusivamente infor- 
mación redundante decidimos, por una parte centrarlo no en la población general, sino en grupos más homogéneos de edad. Según estudios previos es la adolescencia y preadolescencia (Barbadillo Griñán, 1993) uno de los momentos decisivos para la instauración de los hábitos lectores, aunque bien es cierto que estos deben tenerse en cuenta desde los primeros años y duran toda la vida. Para ello, seleccionamos a todo el alumnado de un centro de Málaga capital, perteneciente a $6^{\circ}$ de Primaria y $1^{\circ}$ y $2^{\circ}$ de ESO (lo que antes de la LOGSE se denominaba Ciclo Superior) por ser a partir de esta edad cuando se supone que el automatismo lector está adquirido. El total de chicos y chicas de estos cursos era de 212. Fueron sus respectivas familias (padres, madres o la persona que se encargaba de su cuidado, como podía ser la abuela o la hermana mayor) quienes constituyeron la muestra de este estudio.

\subsection{Las técnicas empleadas}

Aunque el trabajo de campo se inició en el curso 95-96, la recogida de datos en su totalidad se realizó en el curso 97-98. Para ello se han empleado los siguientes procedimientos:

- Entrevistas abiertas y cuestionarios estructurados

- Observación.

A) No participante. Por una parte directa, a través de aquellas formas de investigación sobre el terreno, en contacto con la realidad como son la entrevista y el cuestionario. Por otro lado, indirecta basada en fuentes documentales: prensa local, censo y archivo municipal, documentos cartográficos (mapas de división administrativa, de densidad de población, de redes de comunicaciones,...).

B) Participante. Se llevó a cabo a través del registro de notas de campo en el momento o inmediatamente después de producirse los fenómenos.

\subsection{El trabajo de campo con las familias}

El trabajo de campo se inició con el contacto con el alumnado. A través de ellos/as se les aviso a las familia con una nota en la que se informaba del estudio y del tema. También se les pedía que anotasen en un papel su domicilio particular para poder acudir a sus casas posteriormente. Para la recogida de datos se contactó con trabajadores y trabajadoras sociales que la llevaron a cabo, dada la facilidad de acceso, el desenvolvimiento y el conocimiento que tienen de la familia (en general) y esta zona (en particular).

Con el objeto de uniformizar criterios y explicar la finalidad y metodología del proceso de investigación que iban a realizar, tuvimos constantes reuniones con ellas y ellos antes de empezar el trabajo de campo. Se dedicó una semana para trabajar los contenidos que aparecen en la tabla 1.

El seguimiento del trabajo se realizó concretando sesiones de un día por semana. En ellas se presentaban los resultados de las entrevistas realizadas en un informe escrito. En el caso de no haber podido realizar las entrevistas asignadas en el informe debían aparecer las razones de ello. También se planteaban los problemas surgidos y de forma consensuada se trataron de buscar soluciones.

La entrevista a las familias era abierta; los entrevistadores y las entrevistadoras llevaban un guión con los aspectos más importante que se debían tratar en el desarrollo de la misma. Una vez finalizada se hacía un informe relatando no sólo el contenido de la entrevista basado, en las preguntas que planteaba el guión, sino que además se 
Tabla 1. Contenido del curso preparatorio de trabajadores/as sociales

\section{1.- La entrevista como instrumento de recogida de información:}

- La entrevista. Clases.

- Condiciones formales de la entrevista:

* Preparación.

* Ejecución.

\section{2.- El Fracaso Escolar. Un problema social.}

- Aproximación al concepto de fracaso escolar.

- Fracaso escolar versus inadaptación escolar.

- Raíces del fracaso escolar.

a) Atribución causal.

b) Factores emocionales.

c) Status socioeconómico.

d) Malnutrición infantil.

e) Nuevas tecnologías: TV, Vídeo, ordenador.

f) Sistema educativo:

- Profesor

- Obligatoriedad de la enseñanza.

- Programas educativos.

- Fracaso escolar y problemas lecto-escritores. ¿Una relación causal?

- Intervención desde el trabajo social.

3.- Importancia de los hábitos lectores para el desarrollo académico y profesional.

realizaba una descripción del escenario, de la persona entrevistada y de los materiales culturales que a simple vista aparecían en casa. También se incluían las observaciones personales que cada entrevistador o entrevistadora quería anotar, señalando incluso los acontecimientos que no se comprendían (Taylor y Bogdan, 1985).

\section{Análisis de la información}

Se han utilizado dos procedimientos. Por una parte, un análisis descriptivo de los resultados de los cuestionarios a través del subprograma FRECUENCIES del paquete estadístico SPSS.PC (verión 3.0) y un análisis de contenido de las entrevistas y observaciones.

Para realizar el análisis de contenido, en el que han participado, además de la autora, dos colegas, hemos seguido la siguiente secuencia de trabajo:

1) Lectura, por parte de cada una de las personas participantes en la investigación, del total de la información recogida a través de entrevistas y observaciones.

$2^{\text {}}$ ) Extracción individual de las categorías de análisis.

3ํ) Triangulación de las categorías elaboradas en los pasos anteriores.

4ํ) Elaboración de las categorías definitivas.

5) Codificación de los datos. 
Además, el proceso de análisis se completó con el contraste intermétodos, a través del cual se "trianguló" la información obtenida por los distintos instrumentos. Este proceso de contraste se puede definir como "el uso de dos o más métodos de recogida de datos en el estudio de algún aspecto del comportamiento humano" (Cohen y Manion 1990:,333). Los resultados finales se deducen de los datos procedentes de estos procesos de triangulación. Para facilitar la comprensión de los mismos, se acompañan de fragmentos de las transcripciones de las entrevistas y de las observaciones, así como de gráficos obtenidos de los cuestionarios aplicados.

\section{Resultados}

Del análisis de la información obtenida se puede deducir que las familias que han participado en la investigación están formadas por matrimonios jóvenes que tienen poco tiempo libre, incluso para compartir con sus hijos/as (gráfico 2).

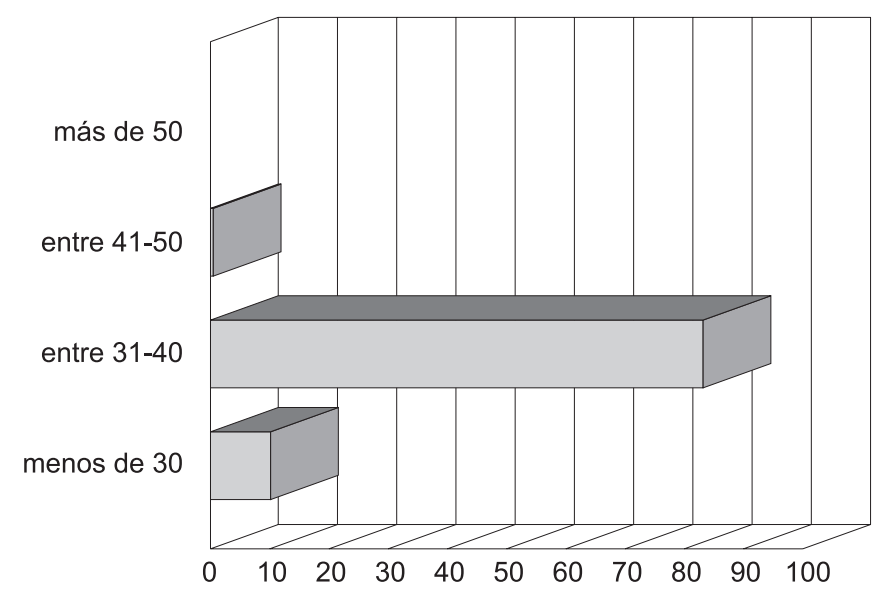

Gráfico 2: Edades de la muestra

Entre sus preocupaciones fundamentales está el trabajo. Los padres trabajan fuera permaneciendo todo el día ausentes del hogar y las madres son las que, en general, se ocupan de sus hijos e hijas, siendo ellas las principales responsables de la familia, de la educación, de las labores del hogar y de las relaciones con el colegio.

\section{"Cuando los llamaban desde el colegio, siempre va la madre" (observación no 144).}

La intensidad y las múltiples responsabilidades siguen siendo las características del trabajo que realizan las mujeres. En aquellos casos en los que las madres trabajan lo hacen también en las profesiones tradicionalmente femeninas (mantenimiento) y compaginándolo con el trabajo en la casa (gráfico 3). En cuanto a las profesiones de los padres suelen ser peones, y aparece un alto porcentaje de ocupaciones indefinidas donde se agrupan todo tipo de actividades relacionadas con la economía sumergida (gráfico 4). 


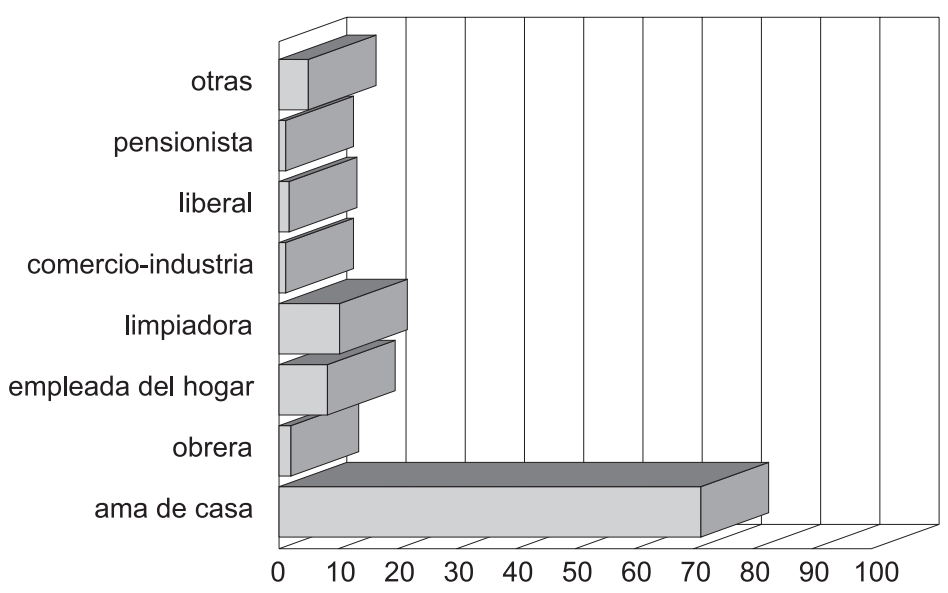

Gráfico 3: Profesiones desempeñadas por las madres

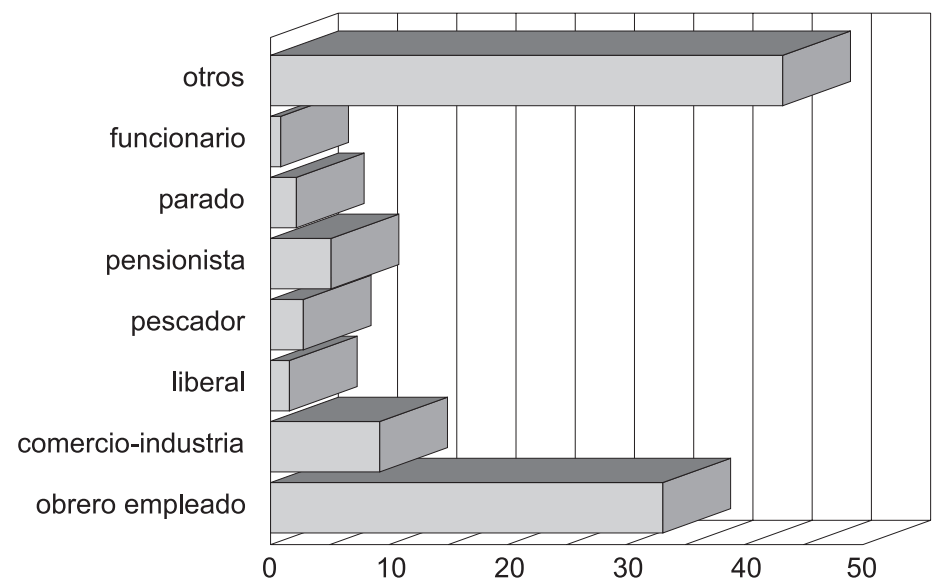

Gráfico 4: Profesiones desempeñadas por los padres

El nivel socioeconómico, en general es bajo. Padres y madres tienen conciencia de esta realidad y la que proyectan en el futuro de sus hijas e hijos. Por ello, parecen conformarse con que estudien un oficio y, sólo si su economía se lo permite, se plantean que puedan estudiar una carrera.

"A su madre le gustaría que hiciera una carrera, si llegado el momento dispone de recursos económicos, aunque aún es pronto para decidirlo" (entrevista $\mathrm{n}^{\mathrm{o}} 88$ )

A pesar de que la mayoría de las viviendas son humildes se encuentran en buen estado, están limpias y ordenadas, pero todas adolecen de espacio, que al ser escaso condiciona bastante la vida familiar y por ende la lectura. 
"La casa es pequeña, para siete personas. No vimos muy bien la decoración del piso, porque nos atendió en la puerta, pero parecía tener un aspecto aceptable" (observación no 13 ).

"Si se me quiere seducir, no hay más que ofrecerme un libro" (www.luismisa.es, 1998:1). Con esta cita comienza una página en internet dedicada a los libros y a todo lo relacionado con ellos. En ella se pone de manifiesto el poder sentimental que pueden llegar a tener los libros si se ha enseñado a valorarlos. Con esta idea y como una forma de conocer si las familias los apreciaban, en principio como un objeto que les interesa poseer, se preguntó acerca del número de libros que tenían en sus casas. Como se observa en el gráfico 5 no son muchos los libros que dicen tener. Cuando se les pregunta por las razones de ello, es decir, ¿por qué no compran más?, la mayoría de las personas encuestadas señalan que "no saben qué libros comprar" (59\%). Otro porcentaje considerable afirma que porque son caros (38,9\%), un 1,1\% que se los compran sus propios hijos/as y un $1 \%$ que porque los niños o niñas no se los piden (gráfico 6). En definitiva, parece que no son un objeto que interese adquirir para la casa. Resulta paradógico que entre las razones más importantes que aducen señalen el elevado precio, cuando en esta zona el promedio mensual que se gasta una familia en alquiler de películas de video es de 5.000 a 6000 pts., como pudimos averiguar a través de una entrevista a un empleado de un videoclub de la zona.

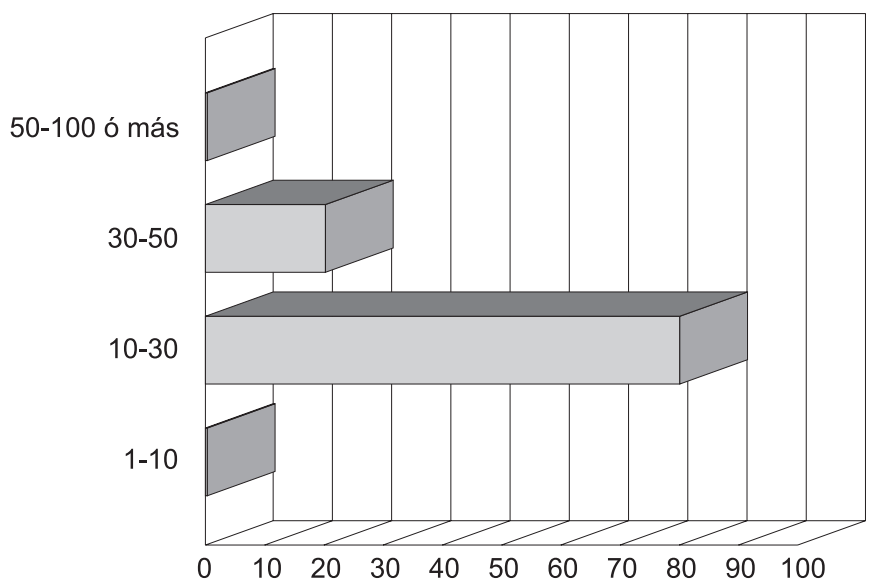

Gráfico 5: Número de libros que tienen en casa

Por una parte, afirman que carecen de información para comprar los libros más adecuados y por otra que no son apropiados los que les mandan en el colegio. Esta aparente incoherencia se debe a que observan que a sus hijos e hijas no les gustan los libros que les mandan. Critican este hecho señalando que además de lo caros que son, no les interesan. Esto contribuye a desprestigiar la lectura tal y como la llevan a cabo en la escuela y el papel que desempeña el profesorado.

Cuando se les pregunta por la temática específica de los libros que tienen en casa, señalan que lo que más tienen son novelas (30\%) y enciclopedias (30\%). En otros casos se 
señalaron los libros de cocina (18\%) y también, aunque en menor proporción, afirman tener de teatro (5\%), poesía (5\%), científicos (7\%) o de historia (7\%) (Gráfico 7). En muchos casos las enciclopedias son los únicos libros que adquieren y puede decirse que no lo hacen voluntariamente, sino porque "se los venden" (generalmente los vendedores acuden a las casas, les dan facilidades de pago, hacen regalos para el hogar por comprarlas, etc.).

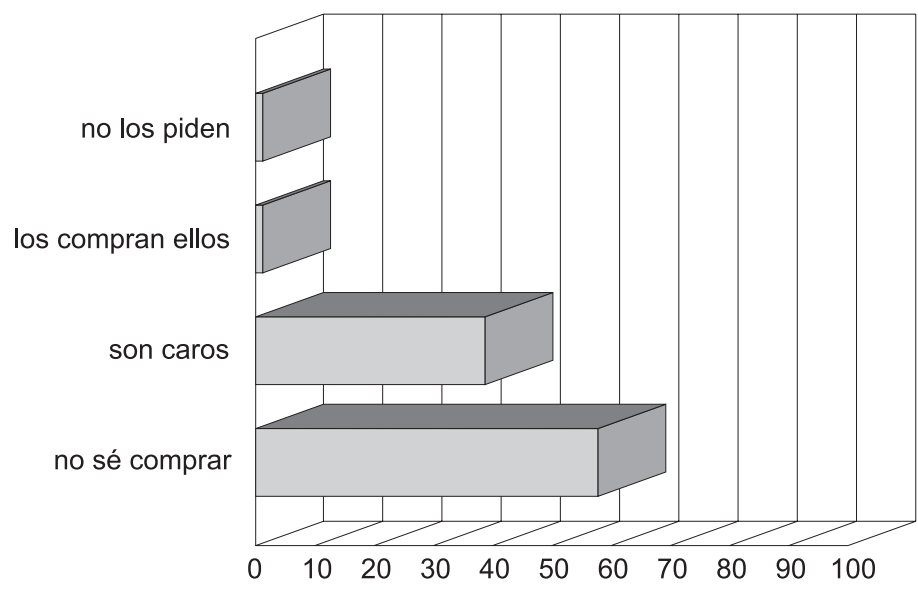

Gráfico 6: Razones para no comprar libros

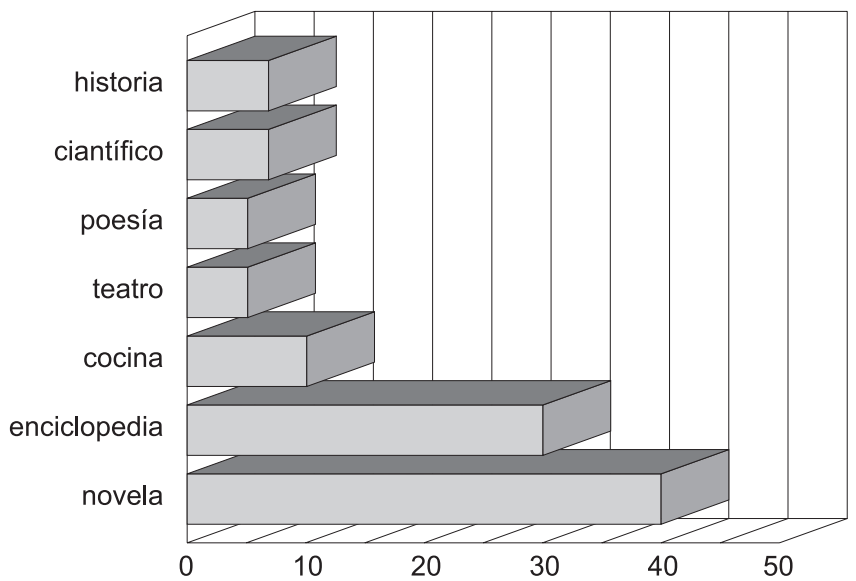

Gráfico 7: Temática de los libros que hay en las casas

"Compran libros del Círculo de lectores porque a su marido le gusta" (entrevista $\mathrm{n}^{\mathrm{o}}$ 90).

Parece que quienes tienen libros en casa lo hacen porque los consideran como adquisición de bienes materiales que son símbolos de la cultura que representa un 
ideal. Procuran tener libros y otros recursos para que los puedan utilizar sus hijos e hijas lo que puede contribuir a mejorar en un futuro su posición social (gráfico 8). De esto se deduce que existe un sentimiento positivo y favorable hacia la formación de sus hijas e hijos y hacia la lectura. Es una actitud que puede dar su fruto creando futuros lectores y lectoras. Siempre que no se conviertan en metros de volúmenes fantasmas que adornan las estanterías (Santos Guerra, 1998).

"Tenía vídeo, radio-cassette, televisión y equipo de música. Tiene un enciclopedia y una colección de libros del Tercer Reich, un curso de inglés con libros y videos" (observación ํo 104).

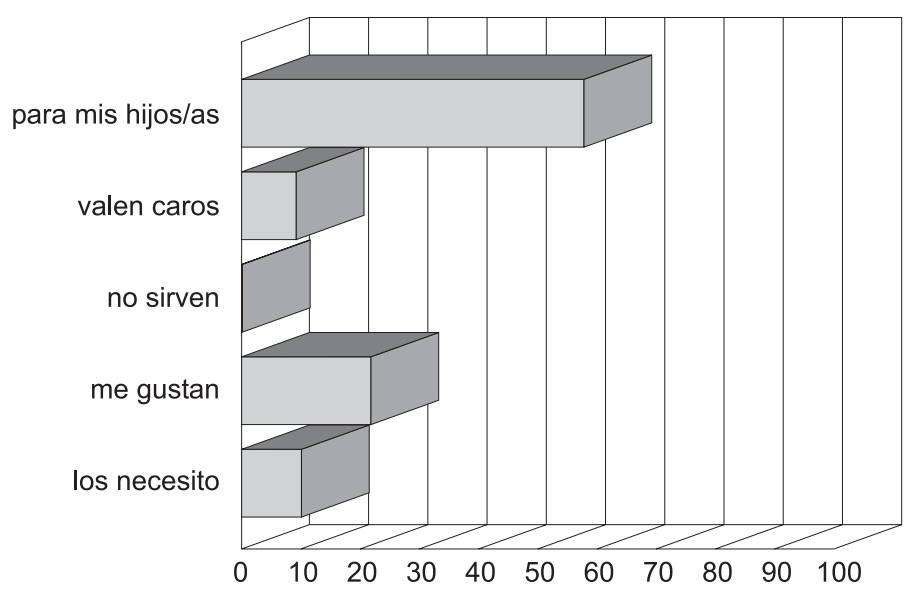

Gráfico 8: Razones para tener libros en casa

Para conocer los hábitos lectores de las familias se les preguntó si leían en casa y en qué momento con el objeto de saber si la lectura era considerada como una actividad cotidiana o excepcional. Aunque hay casos en los que se valora positivamente, no existe un comportamiento lector adecuado en los padres y madres. Un $45 \%$ afirmó que no leían argumentando, como justificación, que era porque trabajaban, destacando con ello el carácter de actividad relacionada con el ocio y el tiempo libre. Otro $45 \%$ afirman que leen cuando tienen un rato libre. Lo que no sabemos es si el primer grupo tampoco lee cuando no está trabajando. Un 11\% señaló que leía con sus hijos/as o cuando les ayudaban en las tareas escolares (Gráfico 9).

Sobre el contenido de la lectura, la mayoría afirma que lee revistas y periódicos (68\%). Sólo un $18 \%$ afirma tener lecturas como poesía, teatro o fantasía. Aquí se pone de manifiesto una vez más la existencia de dos temáticas de lectura y por tanto de dos tipos. De hecho y dado que el porcentaje que afirma que no lee es menor que quienes que leen revistas y periódicos, suponemos que cuando se refieren a estos últimos no los consideran lecturas válidas. El resto reconoce no leer nada (13,1\%) (Gráfico 10). Igualmente ocurre con las de sus hijos e hijas, quienes manifiestan que sus lecturas favoritas y con las que realmente disfrutan son con los tebeos, las revistas juveniles y los libros de terror. 


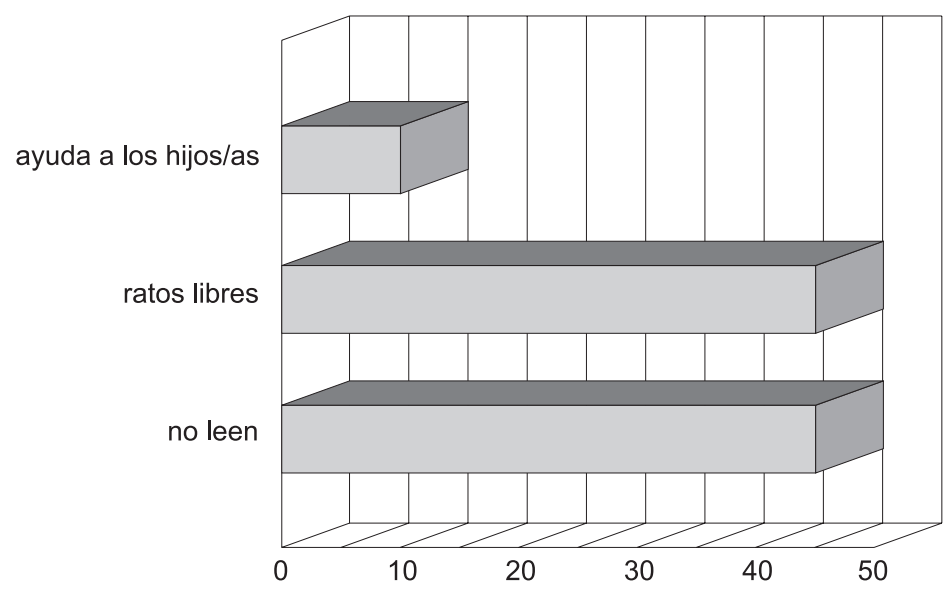

Gráfico 9: Momento de la lectura de padres y madres

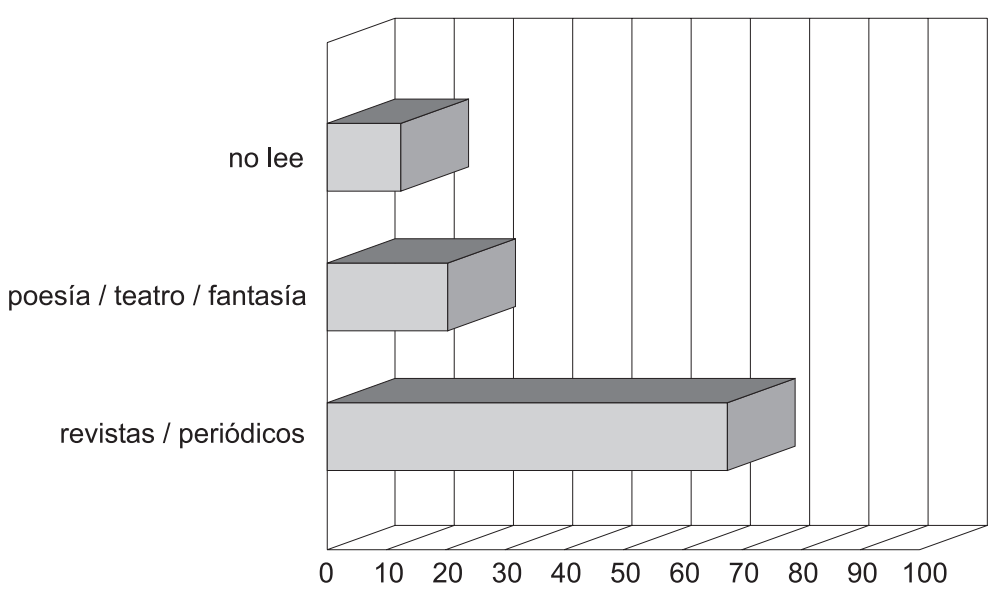

Gráfico 10: Tipo de lectura de padres y madres

Como la lectura por placer no es una actividad habitual es lógico que tampoco exista un lugar específico para que los niños y niñas, ni el resto de la familia, la lleven a cabo y cuando leen lo hacen en cualquier espacio de la casa, especialmente en la habitación y en la cama (un 95,8\%). Probablemente se estén refiriendo en este último caso a la realización de los deberes, ya que la mayoría de las personas identifica la actividad lectora con la realización de las tareas académicas (Gráfico 11).

Reconocen que la lectura es una actividad importante para el presente y el futuro de sus hijos e hijas, pero sus creencias sobre el proceso de aprendizaje, los materiales, las actividades que se pueden realizar sobre ella, etc. están basadas en su experiencia como escolares. 


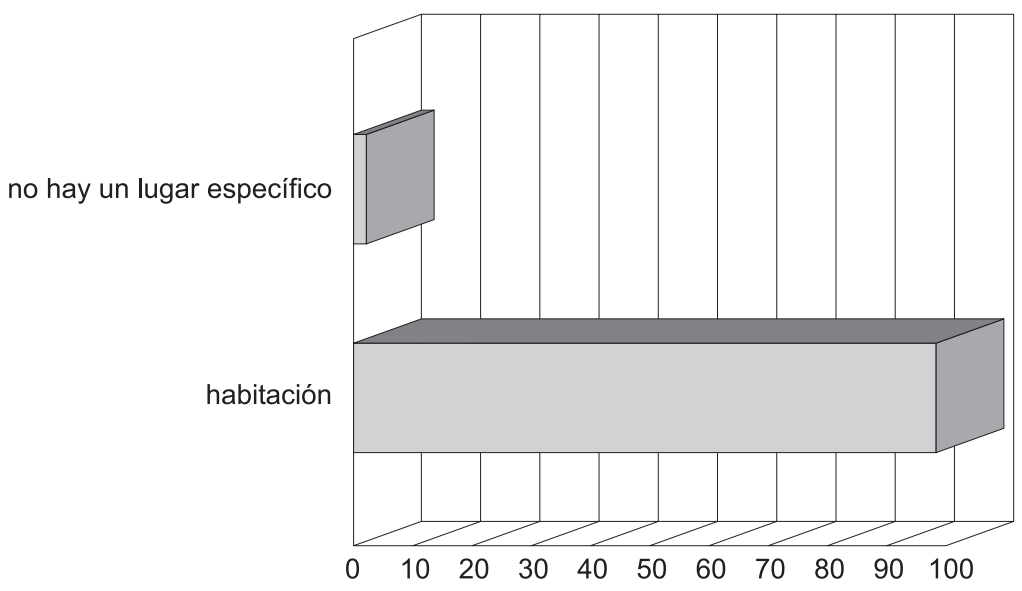

Gráfico 11: Espacio dedicado para leer

La lectura está asociada al descifrado, a los ejercicios de vocabulario, de gramática y al comentario de texto sobre las "grandes obras de la literatura".

En ese sentido, la mayoría de las personas que integran estas familias, no han actualizado su información en torno a la realidad educativa, y ante la evidente desinformación sobre el contexto escolar que denotan, la idea que tienen sobre él es la que permanece en su memoria sobre sus vivencias cuando eran alumnos y alumnas. Para ellos la escuela y los aprendizajes que tienen lugar en ella son sinónimos de obligatoriedad y en muchos casos de castigos. Al igual que Azorín (De Miguel, 1997:83) tienen un amargo recuerdo de su primera escuela:

"Abría yo la cartilla, y durante una hora este maestro feroz me hacía deletrear con insistencia bárbara...Deletreaba una página, me hacía volver atrás, volvíamos a avanzar, volvíamos a retroceder, se indignaba de mi estulticia, exclamaba a grandes voces: ¡Que no!, ique no! Y al fin yo, rendido, anonadado, oprimido, rompía en un largo y amargo llanto".

En "El Florido Pensil" (Sopeña Monsalve, 1994:92) también se recogen innumerables ejemplos de prácticas de este tipo.

"Con las niñas estaba la variante de la muñeca, que me acordaba yo de Valen-ti-na, una niña que salía en mi primera cartilla, que su madre estaba enferma y su hermanito tenía hambre y ella agarraba la muñeca y se la daba a una señora a cambio de pan, que la señora era su salvación; y escribíamos:

Dios no des am pa ra ja más a los bue nos".

Tampoco parece que están muy interesadas en cambiar y en conocer la escuela; van al colegio cuando se les Ilama, cuando sus hijas e hijos tienen algún problema y sólo para asistir a reuniones. Incluso hay casos en los que se desentienden totalmente de todos los temas educativos. Las razones de este hecho hay que buscarlas una 
vez más en la falta de tiempo y en la ausencia de una cultura de participación en los centros escolares.

"La madre sólo va al colegio cuando la llamaba el profesor porque quería hablar con ella. Y tampoco participaba en actividades relacionadas con el colegio" (entrevista no 117).

Se observa una discrepancia entre la familia y la escuela y, aunque tienen intereses comunes, las críticas recíprocas son constantes. Este desconocimiento mutuo unido a la falta de comunicación y en muchos casos de consideración hacia la labor del profesorado, hace que releguen a la escuela todas las cuestiones referidas, no sólo a la lectura, sino a otros aspectos educativos, desentendiéndose de ellos.

"El padre no tenía ni idea del curso que hizo su hijo el año pasado, si repetía o el hace este año, ni al colegio al que va" (entrevista no 6).

Entre los factores que influyen en la lectura consideran que es fundamental el interés de las chicas y chicos (30\%), aunque hay que destacar que también reconocen el papel que puede ejercer la familia (un 20\%) y el profesorado (un 20\%). A este respecto resaltan la importancia de presentar modelos adecuados. Por último, un 10\% considera que es la maduración y un $20 \%$ lo atribuye al método de lectura (gráfico 12).

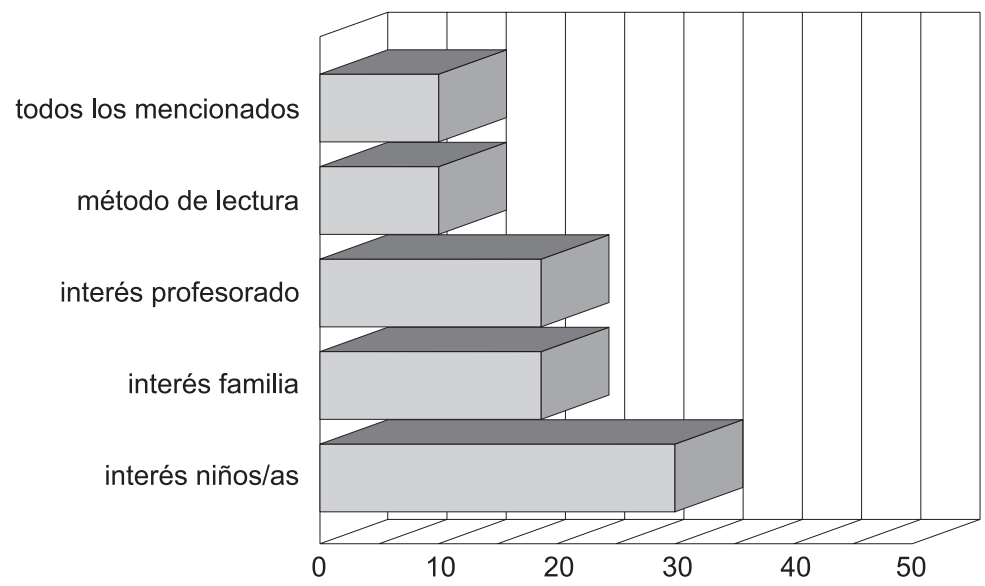

Gráfico 12: Factores que influyen en los hábitos lectores de los/as hijos/as

En general, consideran que sus hijos e hijas no tienen interés por la lectura y a esto hay que unir un desinterés generalizado por lo académico.

"La madre dice que los niños no están interesados en la lectura, que leen bastante poco" (entrevista no 10).

Este es un hecho que les preocupa, destacando como principal inconveniente para los hábitos lectores, en su caso, la falta de tiempo y en el de sus hijos/as la televisión. 
"Uno de los motivos es la entrada de un solo sueldo en la casa (el del padre de familia) que parece ser bajo. Nos dice (la madre) que los libros son muy caros, pero, el motivo principal de que no se lea es la televisión (según la madre de familia). Esta ocupa el ocio de la casa" (entrevista no 2).

De hecho cuando tienen tiempo libre prefieren ver la televisión en lugar de realizar otras actividades (gráfico 13). Así, las referencias al tiempo que los hijos e hijas dedican a la lectura señalan que es los días laborables, donde realizan esta actividad como continuación de las tareas escolares. Parece ser que en vacaciones y días de fiesta prefieren llevar a cabo otras actividades distintas a la lectura.

"Generalmente es al mediodía y después de las clases, por la tarde, cuando se dedica a leer" (entrevista $\mathrm{n}^{\circ} \mathbf{8 2}$ ).

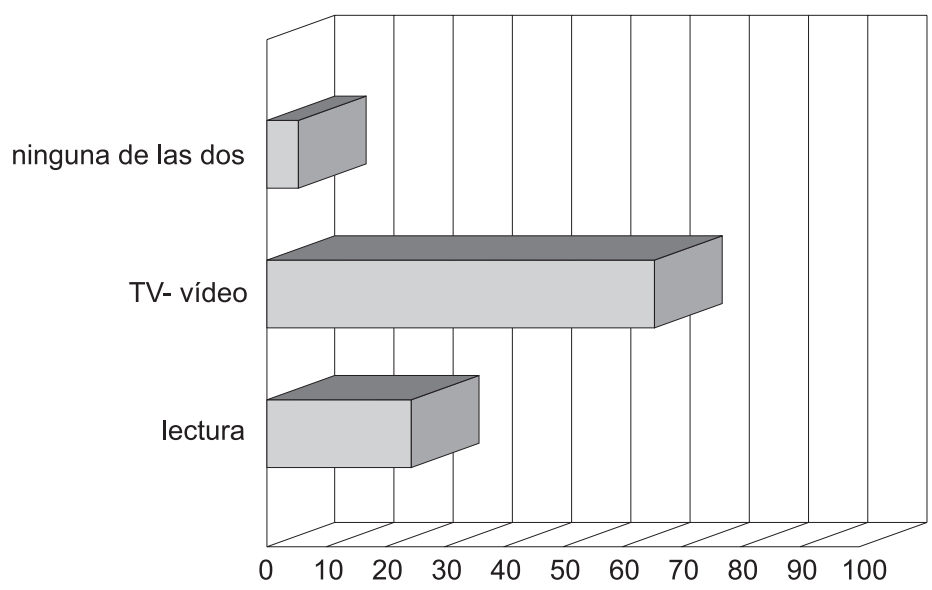

Gráfico 13: Actividad de tiempo libre preferida por los hijos e hijas

Tampoco narran cuentos a sus hijos e hijas ni llevan a cabo otras actividades que les animen a leer, incluso un $60 \%$ afirma que nunca lo ha hecho (gráfico 14). Parecen desconocer que cuando se les cuentan historias y cuentos a las/os niñas/os se está favoreciendo el vínculo entre sus fantasías y sus juegos y los libros. Estos se convierten en el soporte. Siendo la ausencia de estos primeros contactos con la lectura una de las causas de la falta de los hábitos lectores.

"La madre no les leía cuentos a sus hijos de pequeños" (entrevista no 13)

"Justificaba el no contarles historias a sus hijos porque siempre había estado trabajando" (entrevista no 16 ).

Por último, hay que destacar que no perciben las bibliotecas como espacios cercanos a las personas adultas ni a los niños y niñas. Reconocen que sus hijos e hijas acu- 
den a ellas, pero no para divertirse, sino para realizar las tareas del colegio. Una vez más se vincula la lectura en la familia a la obligatoriedad de las actividades escolares.

"Acudía a la biblioteca cuando tenía algún ejercicio de clase que hacer" (entrevista $\mathrm{n}^{\mathrm{o}}$ 76).

La familia tampoco les acompaña y en muchos casos no saben ni siquiera donde están situadas. Algunos padres y madres afirman que su hijo o hija va sólo a la biblioteca del colegio, pero no hacen referencia a que acudan a la biblioteca pública en el barrio.

"En el colegio dedican también tiempo para que los niños vayan a la biblioteca a leer. J.F. lee sobre todo libros de misterio" (entrevista ํo 31).

Todos los factores familiares que se han mencionado tienen un papel fundamental, no sólo en el aprendizaje y en la adquisición de los hábitos lectores de sus hijos e hijas, sino también en su actitud hacia la lectura.

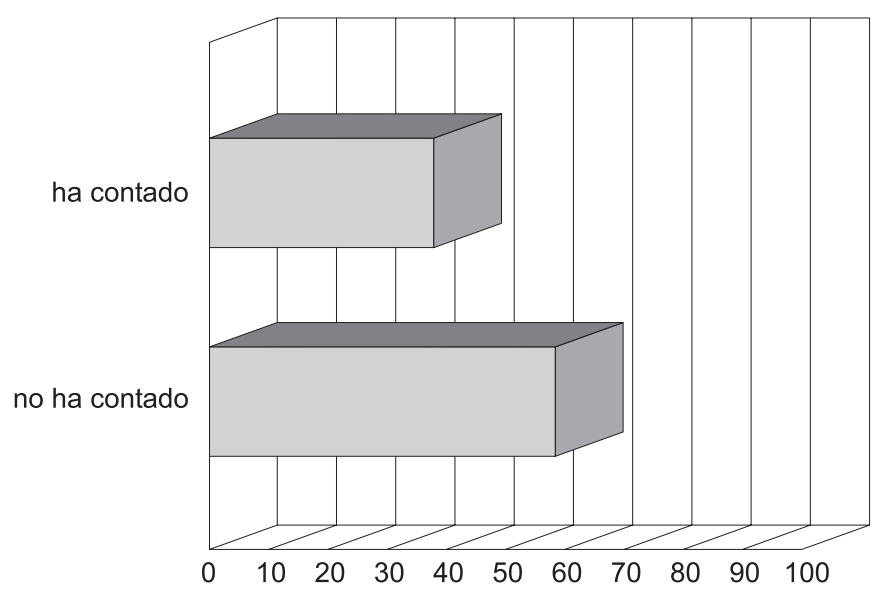

Gráfico 14: Hábito de contar historias y cuentos a los/as hijos/as

\section{Consideraciones finales}

De la información recogida en los diferentes instrumentos acerca de los factores familiares que influyen en la adquisición de los hábitos lectores de las chicas y chicos de 11 a 14 años de un colegio de un barrio de la periferia de Málaga, se pueden extraer las conclusiones que a continuación se señalan, aunque éstas deben calificarse como consideraciones finales, ya que éste es un informe abierto y las conclusiones están presentes en la espiral de comprensión que genera la exploración cualitativa.

Así, de la información obtenida en esta investigación se deduce que existen al menos dos conceptualizaciones de la lectura. Por un lado la de libros y por otro la de tebeos, revistas y periódicos. Esto supone la existencia de dos culturas distintas, valo- 
rándose únicamente la primera y considerando una pérdida de tiempo la segunda. Por ello, aunque se lean, estos materiales no se consideran válidos.

En general, el entorno y los factores familiares no favorecen la adquisición de hábitos lectores. Los padres y las madres no propician las condiciones necesarias para que lean. Carecen de recursos y el material lector que le proporcionan a sus hijos e hijas es, sobre todo, el relacionado con la escuela. Valoran la lectura como algo que les va a permitir mejorar en el futuro a través de los estudios y no como un medio de disfrute. Por lo tanto, los niños y niñas siguen viendo la lectura como una imposición o castigo.

Hay que destacar que las madres y los padres tampoco constituyen modelos válidos y los chicos y chicas siguen viendo que las personas de su entorno no leen; sólo lo hacen ellos y ellas por obligación. Esto provoca que su "norma subjetiva" sea confusa respecto de la lectura, vinculándola como conducta propia a los deberes escolares. De hecho, la familia sólo anima a leer durante el curso académico.

A la vista de los datos, se puede llamar la atención sobre la necesidad de desarrollar actividades de animación a la lectura, no sólo dentro del ámbito escolar, sino especialmente en el seno de la familia. Es en ella, donde los niños y las niñas deben encontrar actitudes favorables hacia los libros, y los modelos y patrones de actuación que conforman su personalidad y su modo de vida. El valor que las madres y los padres le dan a la lectura, sus hábitos lectores y la calidad del material de lectura que manejan está relacionado con las actitudes de los niños y las niñas hacia la lectura. Además, la actitud de una persona hacia la lectura puede influir, a su vez, en su comprensión del texto. Una persona que rechaza la lectura, no llevará a cabo las tareas que esta actividad requiere de manera eficaz, ni placentera. Puede ser que posea las habilidades requeridas para comprender con éxito un texto, pero su predisposición general va a interferir en el uso que haga de esas habilidades (Cooper, 1990).

El conocimiento de los factores analizados es de gran importancia para el diseño de programas de intervención que faciliten la adquisición del lenguaje y la lectura y desarrollar la afición por ésta. En este sentido, las vías fundamentales de intervención se encaminarían, en la línea que señala Barbadillo (1993), en dos direcciones. Por un lado, a definir políticas que favorezcan la comprensión de la familia respecto del valor real que para sus hijos puede representar la lectura, y por otro, al desarrollo, desde los centros educativos, de actividades que sirvan para potenciar en la familia actitudes favorables hacia la lectura.

\section{Referencias Bibliográficas}

ADAN, I. y otros. (1992). Animación. CLIJ, 43, 8-12.

Association Française Pour la Lecture, (1983). Lire, c'est vraiment simple! Paris: Editions MDI.

BARBADILLO GRIÑÁN, P. (1993). La lectura infantil en el ámbito familiar. Infancia y sociedad, 21-22, 138-147.

COHEN, L. y Manion, L. (1990). Métodos de investigación educativa. Madrid: La muralla.

COLEMAN, D. (1996). La inteligencia emocional. Madrid: Kairós.

COOPER, J. D. (1990). Cómo mejorar la comprensión lectora. Madrid: Aprendizaje Visor- MEC. 
CUBERO, R. y MORENO, M. C. (1994). Relaciones sociales: familia, escuela, compañeros. Años escolares. En Palacios, J. Marchesi, C. y Coll,C. (Comp.). Desarrollo Psicológico y educación, I. Madrid: Alianza Editorial.

DE MIGUEL, A. (1997). Autobiografía de los españoles. Así nos vemos, ¿Así somos? Madrid: Planeta.

FOUCAMBERT, J. (1989). Cómo ser lector. Barcelona: Laia.

MILLER, A. And others (1986). Parental participation in paired reading: a controlled study. Educational psycology, 6, 3, 277-84.

MORENO, E., PADILLA, M. y VÉLEZ, E. (1998). La familia como contexto de adquisición de hábitos lectores. Un estudio descriptivo. En Llorent Bedmar, V. (Ed.). Familia y educación. Una perspectiva comparada. Sevilla: Universidad de Sevilla.

PALACIOS, J., MARCHESI, C. COLL, C. (1993). Desarrollo psicológico y educación, I. Madrid: Alianza Psicología.

PANCHÓN IGLESIAS, C. (1995). Intervención con familias en situación de alto riesgo social. CL\&E, 27, 61-74.

POLAINO-LORENTE, A. (1997). Apego y educación temprana. Revista Comunidad Educativa, 244, 11-14.

RODRÍGUEZ, J. M.; MORENO, E. y MUÑOZ, A. (1988). Aprendizaje y ambiente: influencias del entorno familiar y escolar en el aprendizaje de la lectura (I). Revista de Educación Especial, 3, 21-26.

SANTOS GUeRRA, M. A. (1998). Norte del corazón. Málaga: Ed. Málaga Digital.

SILVESTRE, N. y SOLÉ, M.R. (1993). Psicología evolutiva. Infancia, preadolescencia. Barcelona: CEAC.

SOPEÑA MONSALVE, A. (1994). El florido pensil. Memoria de la escuela nacional católica. Barcelona: Grijalbo Mondadori.

TAYLOR, S. y BOGDAN, R. (1985). Introducción a los métodos cualitativos de investigación. Buenos Aires: Paidos.

TOPPING, K. (1986). W.H.I.C.H. Parental involvement reading scheme? Reading, 20 (3) $148-56$.

- (1987). Paired reading: a powerful technique for parent use. Reading teacher, 40 (7) 608-09.

TOPPING, K. y MCKNIGHT, G. (1984). Paired reading-and parent power. Special education: forward trends, 11 (3) 12-15.

VIGOTSKY, L.S. (1978). Mind and society. Cambridge: MA:MIT Press.

WEINBERGER, J. and others (1986). Involving parents in the teaching of reading: will they respond? Reading, 20 (3) 179-83.

WWW.luismisa.es (1998). 